\title{
Proposta de uma Metodologia para um Problema de Roteamento no Transporte de Funcionários de uma Empresa utilizando Vias Principais de Tráfego e Distâncias Reais
}

D.M.B. COSTA, L.V.S. ZAMBONI, Depto de Desenho, Universidade Federal do Paraná, Brasil

M.T.A. STEINER ${ }^{1}$, C. CARNIERI, A.C.L. da SILVA, Depto de Matemática, Universidade Federal do Paraná, Brasil

L.F. NUNES, Depto de Matemática, CEFET-Centro Federal de Educação Tecnológica do Paraná, Cx.P. 19002, 81531-990 Curitiba, Paraná, Brasil.

\begin{abstract}
Resumo. Neste trabalho é apresentada uma proposta para o problema de roteamento de veículos no transporte de funcionários de uma empresa. O objetivo principal é definir as rotas de forma que sejam utilizadas apenas as vias principais de tráfego minimizando, assim, as distâncias a serem percorridas pela frota e o tempo de permanência dos funcionários nos veículos. O estudo foi aplicado a uma empresa situada na região metropolitana da cidade de Curitiba-PR, cujos funcionários, em sua maioria, residem dentro do próprio município.
\end{abstract}

\section{Introdução}

O tratamento dado aos problemas de roteamento de veículos é bastante diversificado, variando não apenas nos algoritmos utilizados, mas também no tipo de tratamento dado às particularidades próprias de cada problema. Bodin et al. [1], fazem uma descrição do problema do roteamento clássico de veículos e apresentam uma coletânea de algoritmos para o seu tratamento, assim como diversas aplicações, como, por exemplo, no transporte escolar. Bowerman et al. [2], Zamboni [16] e Steiner et al. [15] também abordam o problema de roteamento de ônibus escolar urbano. Graciolli [8] faz a abordagem do problema de roteamento na coleta de resíduos sólidos de serviços de saúde. Renz [14] aborda o problema de roteamento com restrições de tempos de viagens e de trabalho para equipes de inventário florestal, responsáveis por coleta sistemática de informações. E, assim, poder-se-ia citar muitos outros

\footnotetext{
${ }^{1}$ tere@mat.ufpr.br
} 
trabalhos de roteamento de veículos, pois este é um dos assuntos mais estudados na área de Pesquisa Operacional (Bodin et al. [1]).

Este trabalho apresenta uma proposta para o problema de roteamento de veículos para o transporte de funcionários de uma empresa utilizando apenas as vias principais de tráfego, sendo que as vias secundárias foram utilizadas apenas nos casos onde havia a necessidade de se fazer a ligação entre as principais. Assim sendo, o funcionário não seria apanhado em sua residência, mas em um ponto de uma via principal o mais próximo possível da mesma. Com este objetivo foi estabelecida uma proposta para a formação dos clusters dos pontos para atendimento, de modo que as rotas se tornem rápidas e eficientes. O estudo foi aplicado a uma empresa situada na região metropolitana da cidade de Curitiba-PR, cujos funcionários, em sua maioria, residem dentro do próprio município.

O trabalho aqui apresentado foi desenvolvido da seguinte forma:

- com a utilização de um mapa digitalizado da cidade foram localizados os diferentes endereços dos funcionários, obtendo-se as coordenadas geográficas destes pontos, denominados de pontos de parada (PP);

- foi realizado o levantamento dos corredores de acesso dentro da cidade (ruas principais, BR, vias rápidas, etc) determinando-se então, um grafo para o problema, cujos nós são os pontos de interseção das vias, denominados de pontos de cruzamentos (PC) e os PP já mencionados e os arcos são as ligações entre estes nós;

- a seguir, foi realizada a designação dos pontos de parada aos pontos de cruzamentos, utilizando-se a distância euclidiana;

- considerando-se, neste momento, apenas os pontos de cruzamentos que possuíam designação, foi realizada a formação dos clusters através da utilização de algoritmos heurísticos, levando-se em conta a demanda de cada ponto e, paralelamente, fazendo-se o controle da capacidade dos veículos;

- finalmente, foram obtidos os trajetos para as rotas, utilizando-se as distâncias euclidianas e as distâncias "quadra a quadra" para as rotas centrais. Para este último caso, utilizou-se informações contidas num banco de dados sobre: distâncias reais, sentidos das ruas, conversões proibidas à esquerda, de modo a tornar a solução aplicável ao problema.

\section{Descrição do Problema}

Grande parte das empresas localizadas na região metropolitana da cidade de $\mathrm{Cu}$ ritiba cujos funcionários residem, em sua maioria, no próprio município, se utilizam de um sistema de transporte terceirizado, onde empresas de transporte especializadas utilizam frotas de ônibus, micro-ônibus e/ou vans para conduzir os empregados até o trabalho, apanhando-os em suas próprias residências ou em pontos pré-determinados, e devolvendo-os ao final do dia.

É notório que um eficiente sistema de transporte, capaz de conduzir os funcionários em segurança, no menor espaço de tempo possível e a um custo mínimo, tem sido preocupação constante de qualquer empresa que tem como filosofia atingir a qualidade total em seus serviços. 
A empresa pesquisada, localizada na Cidade Industrial de Curitiba, em Araucária, apresenta dois grandes grupos de funcionários: os funcionários de horário administrativo e os funcionários de turno. O primeiro grupo trabalha das 7:45 hs. às 16:30 hs. Já o segundo grupo apresenta funcionários divididos em 5 sub-grupos que se revezam em três turnos de oito horas diárias, com início às 7:30 hs., 15:30 hs. e 23:30 hs., já que o trabalho na empresa deve ser contínuo e ininterrupto. Neste trabalho levou-se em consideração apenas os 396 funcionários do grupo administrativo, que atualmente são transportados por 11 veículos.

Considerando que a capacidade máxima permitida dos veículos é de 44 passageiros, utilizou-se neste trabalho exatamente 9 ônibus para efetuar o transporte.

\section{Etapas para o desenvolvimento da solução}

\subsection{Cadastramento dos endereços dos funcionários}

O cadastramento dos funcionários em um mapa digitalizado da cidade de $\mathrm{Cu}$ ritiba, foi feito um a um, utilizando os seus endereços fornecidos pela empresa. Convêm lembrar que se trata de um processo dinâmico, já que são constantes as mudanças de endereços, aposentadorias e contratações de novos funcionários na empresa. Obtiveram-se assim, as coordenadas geográficas dos pontos de parada.

Do total de 396 funcionários, tem-se 20 coincidências de endereços, sendo que tem-se um endereço com 4 funcionários, outro com 3, 15 com 2 funcionários cada e 359 endereços com 1 funcionário, totalizando assim os 396 funcionários localizados em 376 pontos de parada como pode ser visto na Figura 1.

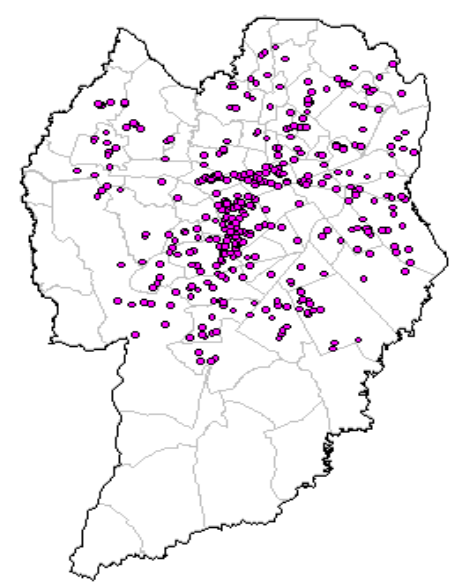

Figura 1: Localização dos pontos de parada (residências dos funcionários) 


\subsection{Cadastramento dos corredores de acesso}

Foi realizado um levantamento dos corredores de acesso dentro da cidade totalizando 71 vias principais, apresentadas na Figura 2. Estas 71 vias principais estão segmentadas, no mapa digitalizado, em 2.128 partes. Ficaram definidos, ao todo, 780 pontos de cruzamentos.

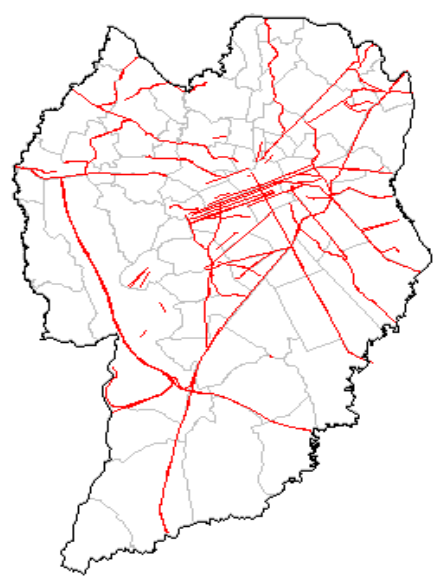

Figura 2: Localização das Vias Principais de Tráfego

Tem-se então um grafo para o problema, onde os nós são os 654 pontos (376 pontos de parada que devem ser designados para os 278 pontos de cruzamento), e os arcos são as ligações entre os pontos de cruzamento.

\subsection{Designação dos pontos de parada aos pontos de cruza- mentos}

Fez-se, nesta etapa, a designação dos pontos de parada aos pontos de cruzamento utilizando-se a distância euclidiana. Dos 278 PC, 210 receberam a designação de 1 $\mathrm{PP}$, outros 40 PC receberam a designação de $2 \mathrm{PP}$ cada um, $16 \mathrm{PC}$ receberam a designação de 3 PP cada, 8 PC receberam a designação de 4 PP, 1 PC recebeu a designação de 5 PP e, finalmente, 3 PC receberam a designação de 7 PP cada um. Tem-se, assim, os $376 \mathrm{PP}$ designados aos $278 \mathrm{PC}$.

Dos funcionários localizados nestes PP, 71\% irá percorrer de 0 a 500 metros; $17 \%$ percorrerá de 500 a 1.000 metros; $11 \%$, de 1.000 a $1.500 \mathrm{~m}$ e $1 \%$, de 1.500 a 2.000 metros, em média, já que foram consideradas as distâncias euclidianas.

\subsection{Formação dos clusters de atendimento}

Como já mencionado, sabe-se que serão necessários 9 veículos com capacidade média de 44 lugares para fazer o atendimento dos 396 funcionários. Deveremos obter, 
portanto, a formação de 9 clusters de atendimento.

Considerando-se apenas os $278 \mathrm{PC}$ que possuem designação, foi realizada a formação dos clusters através da utilização de alguns algoritmos heurísticos, apresentados a seguir, levando-se em conta a demanda de cada ponto de parada.

\subsubsection{Obtenção das Sementes}

O primeiro passo para a obtenção dos clusters é a definição da localização das sementes. Para isto, definiu-se, inicialmente, um ponto fictício (PF), localizado em um ponto da BR277, pelo qual todos os veículos deverão passar para chegar na empresa, que pelo fato de se situar na região metropolitana de Curitiba, não se encontra no mapa apresentado.

Com a definição deste PF, e tendo-se as distâncias entre todos os pontos de parada, $c(i, j)$, obtiveram-se as sementes, conforme o procedimento apresentado a seguir (Paraíba et al. [13]), onde o PF "funcionará" como depósito central.

Passo 1. Faça $\mathrm{k}=1$. Encontre $\mathrm{i}_{k}$ tal que $\mathrm{c}\left(0, \mathrm{i}_{k}\right)=\max \{\mathrm{c}(0, \mathrm{i})\}$, para todo $\mathrm{i}$, onde 0 é o depósito central, ou seja, encontre o ponto de demanda mais distante do depósito central;

Passo 2. Enquanto $1 \leq \mathrm{k}<\mathrm{m}$, faça:

$\mathrm{k}=\mathrm{k}+1$. Encontre o ponto de parada $\mathrm{i}^{*}$ ainda não selecionado tal que:

$\mathrm{c}\left(\mathrm{i}, \mathrm{i}_{k-1}\right)+\mathrm{c}\left(\mathrm{i}, \mathrm{i}_{k-2}\right)+\ldots+\mathrm{c}\left(\mathrm{i}, \mathrm{i}_{2}\right)+\mathrm{c}\left(\mathrm{i}, \mathrm{i}_{1}\right)$ seja máximo para $\mathrm{i}=\mathrm{i}^{*}$.

Faça $i_{k}=i^{*}$.

O algoritmo descrito determina as sementes de forma que estejam o mais longe uma das outras, evitando a concorrência na subseqüente formação dos clusters, como apresentado na Figura 3.

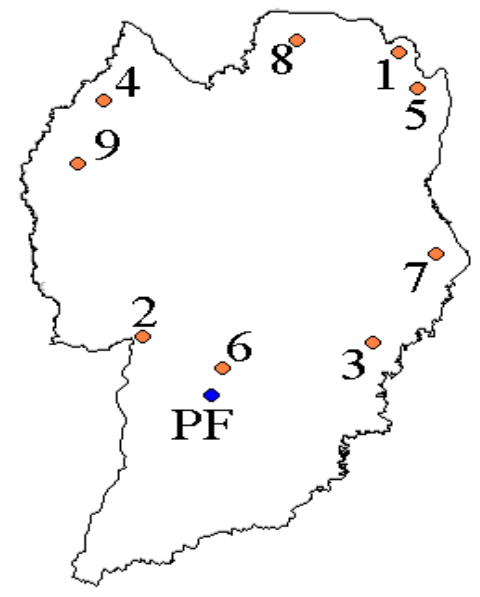

Figura 3: Localização do ponto fictício (PF), comum a todas as rotas, e das 9 sementes 


\subsubsection{Obtenção dos Clusters de atendimento}

Obtidas as sementes, aplicou-se o procedimento proposto por Gillett e Johnson, 1973 (Bodin et al. [1]), (Golden et al. [6]) para a obtenção dos clusters:

Inicialmente, todos os pontos encontram-se sem designação.

Para cada nó i, seja t'(i) a semente mais próxima a i, e t"(i) a segunda semente mais próxima a i.

Para cada nó i, a razão $\mathrm{r}(\mathrm{i})=\mathrm{c}(\mathrm{i}, \mathrm{t}$ '(i) $) / \mathrm{c}(\mathrm{i}, \mathrm{t}$ "(i)) é calculada e todos os nós são colocados em ordem crescente pelos valores de r(i), e assim fica determinada a ordem na qual os nós são designados a uma semente. Aqueles que estão relativamente próximos a uma semente são considerados primeiro, priorizando os PP com demandas maiores. Depois que um certo número de nós foram designados da lista de r(i), um pequeno cluster é formado ao redor de cada semente.

Os nós i cuja razão r(i) está próxima de 1, ou seja, i é um nó que pode ser agrupado por t' ou t", são designados como se segue. Se dois nós adjacentes (vizinhos) $\mathrm{j}$ e $\mathrm{k}$ já estão designados a uma semente, inserindo $\mathrm{i}$ entre $\mathrm{j}$ e $\mathrm{k}$ na rota ligada a $\mathrm{t}$ (t' ou t"), cria um custo adicional igual a $\mathrm{c}(\mathrm{j}, \mathrm{i})+\mathrm{c}(\mathrm{i}, \mathrm{k})-\mathrm{c}(\mathrm{j}, \mathrm{k})$ ao custo total. Ou seja, o algoritmo designa o nó i a uma semente t (t' ou t") inserindo i entre dois nós já designados a semente $\mathrm{t}$ ( $\mathrm{t}$ ' ou t") de maneira que minimize os custos. $\mathrm{O}$ nó $\mathrm{i}$ será designado à semente t ( $\mathrm{t}$ ' ou t") que fornecer o menor custo adicional.

A cada designação de um PP a uma semente, faz-se a verificação da capacidade do veículo. Caso este já esteja com a sua demanda completa, tenta-se fazer a designação para a $2^{a}$ semente mais próxima, sempre seguindo o procedimento anteriormente apresentado.

\subsection{Formação dos roteiros em cada cluster}

Obtidos os clusters ótimos, deve-se obter o roteiro para cada veículo, ou seja, definir a seqüência na qual a coleta dos funcionários deve ser feita, e para isto foi utilizado o algoritmo heurístico de Inserção mais Econômica, descrito a seguir.

\subsubsection{Obtenção dos roteiros}

Para a obtenção da seqüência dos PP em cada cluster, foi aplicado o algoritmo heurístico da inserção mais econômica, devido a Rosenkrantz, Sterns e Lewis, 1977 (Golden et al. [7]), (Bodin et al. [1]), que considera uma rota com k nós na iteração $\mathrm{k}$ e determina qual nó, que ainda não está na rota, poderia ser o próximo a ser ligado à rota (seleção) e então determina onde, na rota, ele deveria ser inserido (inserção). Para isso, são adotados os seguintes passos:

Passo 1. Comece com um sub-grafo consistindo somente do nó i;

Passo 2. Ache um nó $\mathrm{j}$ tal que c(i,j) seja mínimo e forme a sub-rota i-j-i;

Passo 3. Passo da Seleção e Inserção. Para todo arco (i, j) na sub-rota, e k que não esteja na sub-rota, calcule $c(i, k)+c(k, j)-c(i, j)$. Insira $k$ entre $i$ e $j$ que forneça mínimo valor;

Passo 4. Se um circuito Hamiltoniano tiver sido formado, pare. Caso contrário, volte ao Passo 3. 
O algoritmo da inserção mais econômica foi aplicado separadamente para cada um dos clusters formados em 3.4, considerando sempre as distâncias euclidianas entre os pontos de demanda.

Construídos os roteiros para cada um dos ônibus, propõe-se um refinamento baseado em trocas de pontos entre rotas ou ainda, em trocas na seqüência de pontos numa mesma rota de tal forma a obter uma redução do custo total, evitando cruzamentos entre rotas e dentro de uma mesma rota, respectivamente.

\subsubsection{Procedimentos para melhoria do roteiro}

Dos numerosos procedimentos existentes para melhoria de rotas, o presente trabalho faz a abordagem e a aplicação do procedimento de troca de pontos entre duas rotas, descrito por Paraíba [13], e do procedimento das melhorias 2-opt e 3-opt em uma rota, desenvolvido por Lin e Kernighan [11], apresentados a seguir:

Procedimento 1: Troca de pontos entre duas rotas $\mathrm{R}_{s}$ e $\mathrm{R}_{k}$.

Seja $\mathrm{s}_{t} \in \mathrm{R}_{s}$ (localizado entre $\mathrm{s}_{t-1}$ e $\mathrm{s}_{t+1}$ ) e $\mathrm{k}_{m} \in \mathrm{R}_{k}$ (localizado entre $\mathrm{k}_{m-1} \mathrm{e}$ $\left.\mathrm{k}_{m+1}\right)$. Se:

$\mathrm{c}\left(\mathrm{s}_{t-1}, \mathrm{k}_{m}\right)+\mathrm{c}\left(\mathrm{k}_{m}, \mathrm{~s}_{t+1}\right)+\mathrm{c}\left(\mathrm{k}_{m-1}, \mathrm{~s}_{t}\right)+\mathrm{c}\left(\mathrm{s}_{t}, \mathrm{k}_{m+1}\right)<\mathrm{c}\left(\mathrm{s}_{t-1}, \mathrm{~s}_{t}\right)+\mathrm{c}\left(\mathrm{s}_{t}, \mathrm{~s}_{t+1}\right)$

$+\mathrm{c}\left(\mathrm{k}_{m-1}, \mathrm{k}_{m}\right)+\mathrm{c}\left(\mathrm{k}_{m}, \mathrm{k}_{m+1}\right)$,

então troque $\mathrm{s}_{t}$ por $\mathrm{k}_{m}$ na rota $\mathrm{R}_{s}$ e $\mathrm{k}_{m}$ por $\mathrm{s}_{t}$ na rota $\mathrm{R}_{k}$.

Se este procedimento for repetido para todos os pares de pontos, então ele reduz a interseção entre os roteiros.

Procedimento 2: Troca de pontos em uma mesma rota.

Aqui, foram aplicados os procedimentos 2-opt e 3-opt para trocas em uma rota: para um dado $\mathrm{k}$, define-se uma k-troca de uma rota consistindo da deleção de $\mathrm{k}$ arcos de uma rota que serão trocados por $\mathrm{k}$ outros arcos de modo a formar uma nova rota. Uma rota é k-ótima se não for mais possível efetuar trocas para melhorar a distância total viajada. Geralmente o 3-opt se aproxima bastante da solução ótima.

Os Procedimentos 1 e 2 foram aplicados ao problema pesquisado até que não fosse mais possível efetuar trocas entre as rotas e nas rotas.

Para aproximar estas distâncias euclidianas às distâncias reais, utilizou-se coeficientes de correção associados às áreas que contêm as rotas dadas pela distância euclidiana (Novaes [12]). Os fatores de correção $\mathrm{r}_{E}$ permitem relacionar de forma aproximada as distâncias euclidianas $\mathrm{d}_{E}$ com as distâncias reais aproximadas $\mathrm{d}_{r}$, de modo que $\mathrm{d}_{r}=\mathrm{r}_{E} \cdot \mathrm{d}_{E}$. Estes roteiros podem ser visualizados na Figura 4.

\subsubsection{Roteamento "quadra a quadra" - considerando distâncias reais e os sentidos das ruas}

Para os clusters localizados na região central da cidade foram obtidos os roteiros "quadra a quadra", ou seja, levando em consideração os sentidos das vias e conversões proibidas à esquerda, de modo a tornar a solução aplicável ao problema.

Para isto aplicou-se o Algoritmo de Floyd (Christofides [3]) para cada um destes clusters centrais, separadamente, obtendo-se assim a menor distância entre todos os pares de nós considerados em cada cluster. Em seguida, aplicou-se o algoritmo da 
inserção mais econômica, obtendo-se assim as distâncias reais para estes roteiros. Um exemplo de um roteiro central encontra-se na Figura 5.

\section{Obtenção e Análise dos Resultados}

Considerando-se os procedimentos apresentados na seção 3 anterior, obteve-se uma distância total percorrida pela frota da empresa igual a $370,80 \mathrm{~km}$. Esta distância comparada com a distância percorrida pela empresa na época da pesquisa, que era de $401,00 \mathrm{~km}$, nos fornece um percentual de melhoria significativa de aproximadamente 7,5\%((401,00 - 370,80) / 401,00). Esta melhoria foi obtida, principalmente, devido a maneira de se concentrar os pontos de parada, obtendo-se os clusters de forma rápida e eficiente.

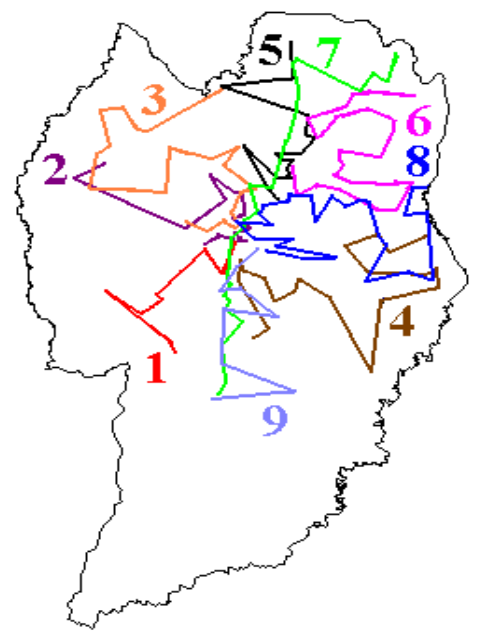

Figura 4: Obtenção dos 9 roteiros a serem percorridos pela frota da empresa

\section{Conclusões}

Este trabalho apresenta a proposta de uma metodologia para o problema de roteamento no transporte de funcionários de uma empresa, utilizando apenas as vias principais de tráfego, e considerando as distâncias reais para as rotas localizadas na região central da cidade de Curitiba.

Esta metodologia utiliza vários procedimentos heurísticos apresentados na literatura que foram adaptados às particularidades apresentadas no problema. $\mathrm{O}$ procedimento é rápido, pelo fato do grafo associado ao problema ser bastante reduzido ao se considerar apenas as vias principais, e eficiente, já que o objetivo é 
fazer com que o funcionário caminhe o mínimo possível até o ponto onde deve ser apanhado.

Poder-se-ia pensar em algoritmos alternativos para solucionar este tipo de problema. Para a obtenção das sementes, por exemplo, poderíamos utilizar o algoritmo das p-medianas. Já para a obtenção dos roteiros, poderíamos fazer uso dos Algoritmos Genéticos (Goldberg [5]), Redes Neurais (Hopfield [9]) ou Simulated Annealing (Kirkpatrick et al. [10]). Por se tratar de procedimentos heurísticos, ter-se-á sempre uma quantidade grande de possíveis soluções.

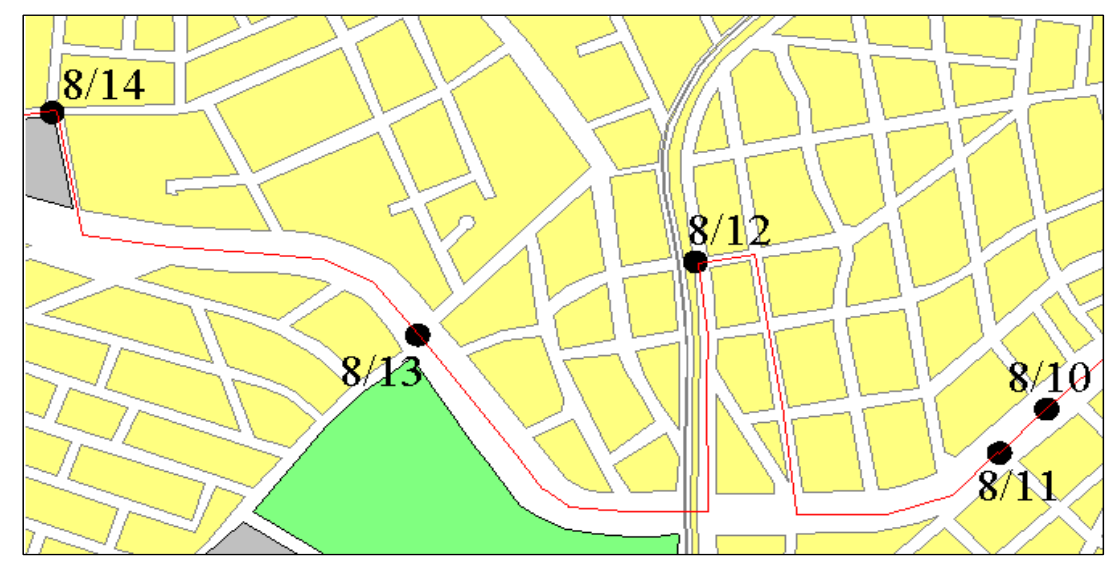

Figura 5: Um exemplo de um roteiro central, considerando o percurso "quadra a quadra"

\section{Referências}

[1] L. Bodin, B. Golden, A. Assad and M. Ball, "Routing and Scheduling of vehicles and crews: the state of the art", England, Pergamon Press, vol. 10, 1983. (Special Issue).

[2] R. Bowerman, B. Hall and P. Calamai, A Multi-Objective Optimization approach to Urban School Bus Routing: Formulation and Solution Method, Transportation Research, 29 No.2 (1995), 107-123.

[3] N. Christofides, "Graph theory - An algorithmic approch", New York, Academic Press Inc., London, 1975.

[4] D.M.B. Costa, Aplicação de algumas Técnicas da Pesquisa Operacional na Otimização dos Serviços Postais, (Dissertação de Mestrado - Métodos Numéricos em Engenharia / Programação Matemática), Universidade Federal do Paraná, 1997. 
[5] D. Goldberg, "Genetic Algorithms in Search - Optimization and Machine Learning, Reading", Addison-Wesley, 1989.

[6] B.L. Golden, T.L. Magnanti and H.Q. Nguyen, Implementing vehicle routing algorithms, Networks, 7 (1977), 113-148.

[7] B. Golden, L. Bodin, T. Doyle and W. Stewart Jr., Approximate Traveling Salesman Algorithms, Operations Research, 28 (3) (1980), 694-711.

[8] O.D. Graciolli, Planejamento de Roteiros de Veículos Coletores de Resíduos Sólidos de Serviços de Saúde, (Dissertação de Mestrado - Engenharia de Produção), Universidade Federal de Santa Catarina, 1994.

[9] J.J. Hopfield, Neurons with graded response have collective computational properties like those of two-state neurons, Proc. Natl. Acad. Sci., (1984), 30883092.

[10] S. Kirkpatrick, C.D. Gelatt Jr. and M. P. Vecchi, Optimization by Simulated Annealing, Science, 220 (1983), 671-680.

[11] S. Lin and B.W. Kernighan, An Effective Heuristic Algorithm for the Traveling Salesman Problem, Operations Research, 21 (1973), 498-516.

[12] A.G. Novaes, "Sistemas Logísticos - Transporte, Armazenagem e Distribuição Física de Produtos", São Paulo, Edgard Bucler Ltda., 1989.

[13] L.C. Paraíba, J.F.R. Fernandes e A.S. Ando, Um Algoritmo Heurístico de Construção Paralela para o Problema do M-Caixeiro Viajante. Trabalho de circulação interna - Departamento de Engenharia Elétrica e Sistemas, UNICAMP, Campinas, 1990.

[14] L.C. Renz, Um Algoritmo para Roteirização com Restrições de Tempos de Viagens e de Trabalho, (Dissertação de Mestrado - Engenharia de Produção), Universidade Federal de Santa Catarina, 1994.

[15] M.T.A. Steiner, D.M.B. Costa, L.V.S. Zamboni, A. LINDBECK da SILVA and C. Carnieri, The Vehicle Routing Problem in the School Transportation, Anais do Congresso, EURO XV / INFORMS XXXIV, Barcelona, Espanha, 1997.

[16] L.V.S. Zamboni, Técnicas de Roteirização de Veículos aplicadas ao Transporte Escolar, (Dissertação de Mestrado - Métodos Numéricos em Engenharia / Programação Matemática), Universidade Federal do Paraná, 1997. 